\title{
Spécificités des urgences toxicologiques pédiatriques
}

\author{
Specificity of pediatric toxicological \\ emergencies
}

\section{Michel LHERMITTE(1), Monique MATHIEU-NOLF ${ }^{(2)}$, Robin CREMER ${ }^{(3)}$, Elisabeth VINNER ${ }^{(1)}$, Damien PEUCELLE ${ }^{(2)}$, Patrick MURA ${ }^{(4)}$, Francis LECLERC ${ }^{(3)}$}

(1) Laboratoire de Biochimie et de Biologie Moléculaire, Hôpital Calmette - 59045 LILLE Cedex

(2) Centre Antipoison,

(3) Service de Réanimation Pédiatrique, Hôpital Jeanne de Flandres, CHRU, avenue de Verdun

59037 LILLE Cedex

(4) Laboratoire de Biochimie et Toxicologie, CHU - BP 577 - 86021 POITIERS Cedex

(Reçu le 10 octobre 2000 ; accepté le 27 octobre 2000)

Chez l'enfant avant l'âge de 12 ans, les intoxications constituent la deuxième cause d'accident à domicile après les traumatismes (1). Elles sont caractérisées par leur grande fréquence et leur habituelle bénignité. Avant l'âge de 6 ans, la bénignité est habituelle ceci s'explique par le caractère accidentel, la faible quantité ingérée, la présence des parents et une consultation médicale rapide. En France, en 1994, on a rapporté 6 décès par divers produits et 9 par le monoxyde de carbone sur les 878 cas d'accidents domestiques dans la tranche d'âge 0-19 ans (2). La stratégie de prise en charge des intoxications aiguës de l'enfant doit tenir compte des données épidémiologiques, différentes de celles des adultes et variable selon l'âge. Elle doit aussi tenir compte des variabilités physiologiques, absorption, métabolisme et excrétion, susceptibles de modifier la cinétique des xénobiotiques $(3,4)$.

\section{Épidémiologie}

La majorité des intoxications concerne l'enfant : 1,08 millions de cas d'ingestion de substances toxiques ont été rapportés en 1998 par les centres antipoison (CAP) américains chez les enfants de moins de 6 ans (5). La proportion des incidents toxiques, rapportés aux CAP américains est estimée à moins de $26 \%$, suggérant un nombre de plus de 4 millions d'intoxications par année aux USA (6). Environ 85000 jeunes enfants ont été traités pour intoxication aiguë en 1998, dans des services d'urgence américains (7). En 1996 les CAP américains rapportaient sur 2,16 millions de cas d'intoxications $59,6 \%$ chez des enfants de moins de 12 ans et $67,1 \%$ des sujets de moins de 20 ans. Les intoxications médicamenteuses représentaient $42 \%$ des appels (5). En France avec environ 100000 cas par an (8), les intoxications représentent la deuxième cause d'accident 
à domicile, derrière les traumatismes (8). De 1995 à 1999, sur les 102567 appels pour intoxication au CAP de Lille, $43 \%$ des appels concernaient des enfants de moins de 15 ans (9).

D'après les données du CAP de Lille de 1995 à 1999 (9) les circonstances d'intoxication varient avec l'âge et les plus fréquentes sont les suivantes:

- pour la classe d'âge de moins de 1 an, $66 \%$ sont liées à un accident domestique, $28 \%$ à une erreur thérapeutique et $4 \%$ à une pollution de l'environnement domestique.

- pour la classe d'âge de 1 an à 4 ans, $94 \%$ sont liées à un accident domestique, et $4 \%$ à une erreur thérapeutique.

- pour la classe d'âge de 5 à 9 ans, $77 \%$ sont liées à un accident domestique, $10 \%$ à une pollution de l'environnement domestique et $9 \%$ à une erreur thérapeutique.

- pour la classe d'âge de 10 à 14 ans, $38 \%$ sont liées à une tentative d'autolyse, $35 \%$ à un accident domestique, $13 \%$ à une pollution de l'environnement domestique et $8 \%$ à une erreur thérapeutique.

- pour la classe d'âge de 14 à 19 ans, $69 \%$ sont liées à une tentative d'autolyse, $13 \%$ à un accident domestique, $6 \%$ à une pollution de l'environnement domestique et $2 \%$ à un accident professionnel ou une toxicomanie.

Au niveau de l'âge, il existe deux pics de fréquence. Le premier se situe entre 1 et 4 ans et correspond aux intoxications accidentelles. Le second est observé à l'adolescence et correspond à des intoxications volontaires.

Les intoxications accidentelles représentent la grande majorité des cas, elles sont plus fréquentes chez le garçon (55\%), ont lieu à domicile (relâchement de la surveillance) et plus spécialement pendant le week-end ou les vacances. $75 \%$ des intoxications se produisent en présence des parents (8), au moment où les enfants ont faim et où les adultes prennent leurs médicaments (8). Le toxique en cause se trouve presque toujours à portée de main de l'enfant et l'accident survient en quelques secondes (10).Les intoxications se produisent par ordre de fréquence décroissante dans la cuisine, la salle de bain, le jardin, la chambre de l'enfant ou des parents (10).

Les intoxications intentionnelles (empoisonnement) par maltraitance sont plus rares, mais leur fréquence est probablement sous estimée en raison des difficultés de diagnostic. La moyenne d'âge est de deux ans, mais tous les enfants peuvent être concernés (11). La suspicion d'empoisonnement repose sur l'existence de discordances à l'interrogatoire des parents, quant aux circonstances de "l'accident". Les signes évocateurs sont rapportés dans le Tableau I (10). L'empoisonnement est un évènement unique dans $70 \%$ des cas (12), il peut être chronique, s'intégrant dans le syndrome de Münchhausen par procuration (12). Les toxiques en cause sont très variés : médicaments (psychotropes), produits ménagers, agricoles (pesticides), alimentaires (eau, sel,..) (10). Ils ne sont pas toujours détectés par les examens toxicologiques de routine. En Angleterre et en Irlande, de 1992 à 1994, 44 cas d'intoxications intentionnelles ont été détectés : dans 28 cas, il s'agissait d'un syndrome de Münchhausen par procuration, les toxiques en cause étant le plus souvent des anticonvulsivants ou des opiacés (13).

A l'adolescence, les tentatives de suicide représentent la majorité des intoxications et les médicaments 65 à $86 \%$ des substances en cause (14-16). En France, il s'agit de psychotropes (14) et dans les pays anglosaxons du paracétamol $(15,16)$. L'ingestion a lieu dans $80 \%$ des cas, au domicile familial (15) et est associée à la prise d'alcool dans $25 \%$ des cas (17). De 1995 à 1999, pour la tranche d'âge 10-19 ans, les tentatives de suicide représentent $53 \%$ des appels au Centre Antipoison, avec une prédominance féminine (10). Ces intoxications volontaires ont un meilleur pronostic que

Tableau I : Signes évocateurs d'un empoisonnement (10).

\begin{tabular}{|l|l|}
\hline \multicolumn{1}{|c|}{ Antécédents personnels et familiaux } & \multicolumn{1}{c|}{ Épisode en cause } \\
\hline Maltraitance & Age inférieur à 18 mois \\
\hline Décès suspects dans la fratrie & Consultation tardive \\
\hline Intoxications répétées & Discordance à l'interrogation des parents \\
\hline Syndrome de Münchhausen & Retard de croissance \\
\hline $\begin{array}{l}\text { Signes cliniques inexpliqués disparaissant lors } \\
\text { des hospitalisations }\end{array}$ & $\begin{array}{l}\text { Signes de maltraitance physique } \\
\text { Signes d'intoxication ou de sevrage aux stupéfiants }\end{array}$ \\
\hline
\end{tabular}


celles de l'adulte, car elles sont souvent des appels au secours sans réel désir de mort (10). La gravité croit cependant avec l'âge (15) et les intoxications volontaires sont plus graves chez les garçons que chez les filles, les médicaments ingérés étant plus toxiques (16). L'entretien avec un psychiatre est nécessaire avant la sortie de l'hôpital de tout adolescent intoxiqué volontaire (10).

A l'adolescence la consommation de substances licites (tabac, alcool), illicites (stupéfiants) ou détournées de leur usage (médicaments, solvants) n'est pas exceptionnelle. Aux Etats-Unis, ce phénomène pose un véritable problème de santé publique et fait l'objet d'enquêtes épidémiologiques. En 1993, 35,3\% des élèves de terminale disaient avoir consommé au moins une fois de la marijuana, $17,4 \%$ avoir inhalé des solvants, $10,3 \%$ avoir consommé du LSD, $6,1 \%$ de la cocaïne et $1,1 \%$ de l'héroïne (17). Chez les enfants de $4^{\text {ime }}$, la prévalence était de $12,6 \%$ pour la marijuana, $19,4 \%$ pour les solvants, $3,5 \%$ pour le LSD, $2,9 \%$ pour la cocaïne et 1,4\% pour l'héroïne (18). En France, la prévalence de la consommation de ces substances n'est pas connue. Même si la dépendance aux substances psychoactives n'a pas la même ampleur qu'aux Etats-Unis, il ne faut pas exclure la possibilité de ce type d'intoxication chez l'enfant jeune. La constatation de troubles du comportement, surtout s'ils sont accompagnés de troubles digestifs ou de troubles hémodynamiques doit évoquer la prise de substances psychoanaleptiques; une rougeur conjonctivale doit évoquer la prise de cannabis, alors que la présence d'une mydriase est constatée $60 \mathrm{~min}$ après prise de LSD.

La recherche de ces substances est rendue, la plupart du temps, difficile du fait de leur demi-vie brève; aussi l'analyse toxicologie des urines est préférable à celle du sang. La caféine est souvent un adultérant de ces substances. Aussi, la présence inexpliquée de caféine doitelle être un argument pour effectuer des recherches toxicologiques approfondies (10).

\section{Gravité des intoxications de l'enfant}

Dans la grande majorité des cas, l'intoxication est bénigne. De 1995 à 1999, $70 \%$ des appels au Centre Antipoison de Lille ne donneront pas lieu à une hospitalisation. L'appel donnait lieu dans $62 \%$ à un simple conseil et un suivi téléphonique par le médecin du Centre Antipoison, dans $8 \%$ à une consultation chez un médecin généraliste ou un spécialiste (ophtalmologue) dans $19 \%$ à une orientation vers un service d'ur- gence, dans $9 \%$ à une hospitalisation en pédiatrie et dans $2 \%$ à une hospitalisation en réanimation pédiatrique ou au centre d'oxygénothérapie hyperbare. Dans $2 \%$ des hospitalisations, le Centre Antipoison de Lille recommandait un transport médicalisé et basculait immédiatement l'appel vers le 15 du département correspondant. Le Tableau II montre la durée moyenne de séjour des enfants pris en charge par le service de Réanimation Pédiatrique du CHRU de Lille en 1995. En France, en 1993, parmi les enfants de moins de 19 ans décédés par accident (homicides et suicides exclus), 7 sont décédés d'intoxication au $\mathrm{CO}$ et 7 d'une autre intoxication (2).

\section{Produits en cause}

En 1994, les intoxications non-médicamenteuses représentaient $63 \%$ des appels aux CAP américains. pour les sujets de moins de 19 ans et les intoxications médicamenteuses $37 \%$ (5). Les produits responsables de ces intoxications sont répertoriés dans le Tableau III. En ce qui concerne les produits non-médicamenteux, il s'agissait de produits alimentaires $(19,7 \%)$, de cosmétiques $(14,3 \%)$, de produits ménagers $(13,8 \%)$, de plantes $(9,2 \%)$, d'hydrocarbures $(3,8 \%)$ et de pesticides $(3,5 \%)$. Parmi les médicaments, on trouvait, les antalgiques (21\%), les antitussifs et apparentés $(15,1$ $\%)$, les topiques cutanés $(10,6 \%)$, les antibiotiques et les antiparasitaires $(8,2 \%)$, et les médicaments gastrointestinaux (7,2 \%). Entre 1995 et 1998, les cosmétiques, les produits de nettoyage, les analgésiques et les antitussifs représentaient $58 \%$ des produits à l'origine d'intoxications chez l'enfant (5).

En 1998, Lutovitz et Coll (5) ont rapporté les substances les plus fréquemment rencontrées dans les intoxications aiguës chez l'enfant âgé de moins de 6 ans. En ce qui concerne les produits non médicamenteux, il s'agissait des cosmétiques et des produits de soins $(13,3 \%)$, des produits ménagers $(11 \%)$, de plantes $(7,1 \%)$, des pesticides $(3,9 \%)$, des hydrocarbures $(2,2 \%)$.

En ce qui concerne les médicaments, on retrouvait les analgésiques $(7,6 \%)$, les antitussifs et apparentés $(5,5 \%)$, des topiques cutanés $(5,4 \%)$, des vitamines $(3,3 \%)$, des antibiotiques $(3,1 \%)$, des médicaments gastro-intestinaux (3\%), des antihistaminiques $(1,9 \%)$ et des hormones ou contraceptifs oraux $(1,9 \%)$.

De 1995 à 1999, les intoxications non-médicamenteuses représentaient $58 \%$ des appels au CAP de Lille, pour les sujets de moins de 19 ans et les intoxications médicamenteuses $42 \%$ (9). Les produits responsables de ces intoxications sont répertoriés dans le Tableau III. En ce qui concerne les produits non-médicamenteux, il 
Tableau II : Durée de la prise en charge des enfants intoxiqués (à l'exclusion des intoxications au CO) admis dans le service de réanimation pédiatrique de Lille en 1995 (10).

\begin{tabular}{|l|c|c|c|c|}
\hline \multirow{2}{*}{} & \multicolumn{2}{|c|}{ Hôpital de jour } & \multicolumn{2}{c|}{ Hospitalisation } \\
\cline { 2 - 5 } & Nombre & Durée moyenne (h) & Nombre & Durée moyenne (h) \\
\hline Intoxications médicamenteuses & 93 & 9 & 18 & 47 \\
\hline Autres intoxications & 61 & 7 & 6 & 86 \\
\hline Total & 151 & 8 & 24 & 56 \\
\hline
\end{tabular}

Tableau III : Produits à l'origine des appels reçus dans les centre antipoisons américains et au CAP de Lille.

\begin{tabular}{|c|c|c|}
\hline TYPE DE PRODUIT & CAP AMÉRICAIN & CAP LILLE \\
\hline AGE INFÉRIEUR A 19 ANS & 1994 (5) & $\mathbf{1 9 9 5 - 1 9 9 9}(\mathbf{9})$ \\
\hline Produits non médicamenteux & $\mathbf{6 3} \%$ & $\mathbf{5 8 \%}$ \\
Produits alimentaires & $19,6 \%$ & $2,8 \%$ \\
Cosmétiques & $14,3 \%$ & $11,1 \%$ \\
Produits ménagers & $13,8 \%$ & $32,2 \%$ \\
Plantes & $9,2 \%$ & $10,0 \%$ \\
Hydrocarbures & $3,8 \%$ & - \\
Pesticides & $3,5 \%$ & $7,9 \%$ \\
Produits de bricolage & - & $11,6 \%$ \\
Gaz, fumées dont CO & - & $7,4 \%$ \\
Alcool & & $0,5 \%$ \\
Stupéfiants, drogue & & $0,4 \%$ \\
\hline Antalgiques & $\mathbf{3 7} \%$ & $\mathbf{4 2} \%$ \\
Antitussifs et apparentés & $21,0 \%$ & $10,3 \%$ \\
Topiques cutanés & $15,1 \%$ & $6,7 \%$ \\
Antibiotiques et antiparasitaires & $10,6 \%$ & $6,5 \%$ \\
Médicaments gastro-intestinaux & $8,2 \%$ & $5,6 \%$ \\
Contraceptifs oraux & $7,2 \%$ & $4,6 \%$ \\
Fluor & - & $10,1 \%$ \\
Hypnotiques, sédatifs & - & $5,9 \%$ \\
Vitamines et fer & - & $5,0 \%$ \\
Médicaments & $1,7 \%$ \\
\hline
\end{tabular}

s'agissait de produits ménagers $(32,2 \%)$, de produits de bricolage $(11,6 \%)$, de cosmétiques $(11,1 \%)$, de pesticides $(7,9 \%)$, de gaz, fumées dont le CO $(7,4 \%)$. Parmi les médicaments, on trouvait les antalgiques $(10,3 \%)$, les contraceptifs oraux $(10,1 \%)$, les antitussifs $(6,7 \%)$, les topiques cutanés (6,5\%) (9).

De 1995 à 1999, le Centre Antipoison de Lille a suivi 39106 cas d'intoxication, $97 \%$ des cas (38 110) n'ont présenté aucun symptôme ou des symptômes de faible gravité, $3 \%$ (996) des intoxications ont été graves avec survenue de 23 décès (Tableau IV).
Les données des services de réanimation pédiatrique diffèrent des données des CAP et font apparaître un taux important d'intoxications médicamenteuses chez les enfants nécessitant une hospitalisation. De 1986 à 1991, parmi les enfants admis dans le service de réanimation pédiatrique du CHRU de Lille, le toxique en cause était un médicament dans plus de $30 \%$ des cas et un produit ménager dans $30 \%$ des cas (10). 
Tableau IV : DÉCÈS (données Centre Antipoison de Lille 1995-1999).

\begin{tabular}{|c|c|c|}
\hline Monoxyde de Carbone & 2 & 3 et 14 ans \\
\hline $\begin{array}{l}\text { Monoxyde de Carbone + Fumées (Incendie) } \\
\text { Dont } 4 \text { avec présence de cyanures associés }\end{array}$ & 15 & 2 à 14 ans \\
\hline Fonzylane & 1 & $\begin{array}{l}\text { Fille } 13 \text { ans } \\
\text { T.A. }\end{array}$ \\
\hline Antidépresseurs tricycliques + Benzodiazépines + Barbituriques lents & 1 & $\begin{array}{l}\text { Fille } 14 \text { ans } \\
\text { T.A. }\end{array}$ \\
\hline Trichloréthylène & 1 & $\begin{array}{l}\text { Garçon } 9 \text { ans } \\
\text { T.A. } \\
\end{array}$ \\
\hline Essence & 1 & $\begin{array}{l}\text { Garçon } 13 \text { ans } \\
\text { T.A. }\end{array}$ \\
\hline Xylocaïne visqueuse & 1 & $\begin{array}{l}\text { Fille } 18 \text { mois } \\
\text { Acc. Domestique }\end{array}$ \\
\hline Paracétamol + plaquenil & 1 & $\begin{array}{l}\text { Fille } 13 \text { ans } \\
\text { T.A. }\end{array}$ \\
\hline
\end{tabular}

\section{Particularités pédiatriques du diagnostic}

Souvent le diagnostic est facilité par la présence d'un témoin (1); il peut être difficile en son absence ou en cas d'intoxication dissimulée. Il faut signaler que, pour les médicaments déconditionnés, les CAP de Nancy et de Lille disposent d'une base informatique capable d'identifier un médicament à partir de sa forme galénique (19). Le diagnostic doit être évoqué devant tout symptomatologie aiguë, non expliquée chez un enfant jusque-là bien portant $(90 \%$ des ataxies aiguës de l'enfant sont dues à des intoxications par les benzodiazépines. Les signes cliniques d'orientation sont les mêmes que chez l'adulte (Tableau V).

Tableau $V$ : Principaux signes cliniques d'orientation devant une intoxication (10).

\begin{tabular}{|c|c|}
\hline Myosis & Troubles du rythme \\
Morphiniques & Digitaliques \\
Mydriase & Tricycliques \\
Atropine & Vomissements \\
Tricycliques & Théophylline \\
Convulsions & Digitaliques \\
Tricycliques & Hyperpnée d'acidose \\
Théophylline & Ethylène glycol \\
Ataxie & Propylène glycol \\
Benzodiazépines & Salicylés \\
\hline
\end{tabular}

\section{Analyses toxicologiques}

Les recherches toxicologiques ne doivent pas être réalisées de façon systématique, mais lorsque l'identification du ou des toxiques est utile au diagnostic ou au traitement (20). Elles seront effectuées sur le liquide gastrique, le sérum $(10 \mathrm{~mL})$ ou les urines $(50 \mathrm{~mL})$. La concentration élevée du toxique dans le liquide gastrique en phase de résorption rend la détection facile, sans être corrélée avec le taux sanguin. La détermination du taux plasmatique ou sérique est importante quand l'attitude thérapeutique en dépend [nomogramme pour le paracétamol (21), indication d'une épuration extra rénale pour le méthanol ou l'éthylène glycol] et dans un but médico-légal.

Les recherches toxicologiques qualitatives classiques font appel à des méthodes colorimétriques [Cyanures $(22), \ldots$ ] enzymatiques [inhibition des cholinestérases par les organophosphorés,.(23)], immunochimiques (24) [recherche de benzodiazépines, de barbituriques, d'antidépresseurs dans le sang, ou recherche de stupéfiants dans les urines]. Les méthodes immunoenzymatiques ne reconnaissent pas toutes les molécules d'une même classe thérapeutique, et d'autre part la réponse dépend de la molécule à rechercher (taux de réaction croisée variant d'une molécule à l'autre dans une même classe thérapeutique) ce qui fait que le seuil de positivité varie d'une molécule à une autre. Toujours en raison de ces réactivités croisées, plus ou moins importantes selon les substances, le risque de 
résultat faussement positif doit toujours être présent à l'esprit. Chez le nouveau-né, une attention toute particulière doit être observée dans le cas du dosage de la digoxine, du fait que le nouveau-né sécrète une substance "digoxine-like", pouvant induire des résultats faussement positifs avec les méthodes immunologiques. La chromatographie en phase gazeuse couplée ou non à la spectrométrie de masse ou la chromatographie liquide de haute performance, couplée à un détecteur à barettes de diodes ou à la spectrométrie de masse sont actuellement très utilisées pour rechercher et identifier de nombreuses molécules organiques $(25,26)$. Ces méthodologiques permettent de plus de procéder à des recherches larges. Dans le domaine des échantillons pédiatriques dont le volume peut être très faible, l'électrophorèse capillaire semble être vouée à un bel avenir (27). Les métaux peuvent être recherchées et dosés par spectrophotométrie d'absorption atomique ou par la torche à plasma (ICP) (28).

Différentes études ont montré l'intérêt de l'analyse toxicologique chez des enfants suspects d'intoxication.

Ainsi, Belson et coll. (26) ont rapporté une étude portant sur 227 sujets âgés de moins de 18 ans. Pour deux tiers des sujets admis à l'hôpital, l'intoxication était connue et la recherche de toxiques n'était effectuée que pour confirmer la prise de la substance. Cependant, cette étude montrait l'importance de l'analyse sérique comparée aux recherches effectuées sur l'urine, le dosage sanguin pouvant définir, dans certains circonstances, la sévérité de l'intoxication et être une indication pour un traitement spécifique.

Dans cette étude, il était également observé que l'attitude thérapeutique changeait pour $10 \%$ des sujets, après un résultat négatif, comparé à $50 \%$ pour un résultat positif.

Il est également important d'effectuer des recherches pour trouver des associations avec d'autres xénobiotiques (26), pour les sujets présentant des déficiences mentales ou des troubles de conscience d'origine inconnue (26). Toutefois, il faut tenir compte, pour ces recherches, du coût des analyses.

Des quantifications de xénobiotiques apparaissent importantes dans certaines intoxications (paracétamol, fer,...) et ceci pour plusieurs raisons : déterminer des concentrations toxiques, prévoir une toxicité retardée possible, évaluer l'efficacité de la thérapeutique.

Chez l'enfant, une attention toute particulière doit être portée sur le paracétamol. La présence de ce principe actif dans de très nombreuses spécialités pharmaceutiques à visée antalgique non soumises à prescription fait que ce produit "inonde" bien souvent les foyers, de manière parfois très accessible. Il constitue donc une cause fréquente d'intoxication involontaire. De plus, certains parents, croyant qu'il s'agit d'une substance dénuée de toute toxicité, n'hésitent pas à donner des doses répétées et importantes (29). Jusqu'à 12 à $24 \mathrm{~h}$ après ingestion, moment à partir duquel est observée une augmentation des transaminases, la symptomatologie est souvent très discrète (asymptomatique ou nausées, vomissements). Tous ces éléments plaident en faveur d'une recherche fréquente de ce produit, même en l'absence de signes cliniques évocateurs. Lors d'une étude réalisée sur 839 enfants décédés dans la région de St Louis (USA) pour des causes diverses, Hanzlick a montré que chez 33 enfants de moins de 5 ans (4\% de la population totale) pour lesquels les décès n'avaient pas été attribués à une origine toxique, le paracétamol se retrouvait à une concentration de $9,9 \mu \mathrm{g} / \mathrm{mL}$, avec des valeur allant de 1 à $34,5 \mu \mathrm{g} / \mathrm{mL}$ (30).

Mahoney et coll. (31) individualisaient 4 groupes de sujets en fonction des résultats toxicologiques :

1) le taux sérique va prédire la toxicité et la thérapeutique est mise en oeuvre en fonction des taux de toxiques mesurés (cas du paracétamol, de la digoxine, de la théophylline),

2) la toxicité est corrélée au taux sérique, mais le traitement ne dépend pas du taux toxique (éthanol),

3) la toxicité et la thérapeutique dépendent des symptômes cliniques; dans ce cas, la recherche toxicologique ne sert qu'à confirmer la présence d'une substance (narcotiques),

4) la toxicité n'est pas corrélée avec le taux sérique et seulement un traitement symptomatique est mise en oeuvre.

D'autre part, Sugarman et coll. (20) considéraient que les recherches toxicologiques étaient utiles dans les circonstances suivantes :

1) confirmer l'ingestion d'une substance connue ou inconnue quand l'examen clinique est anormal,

2) détecter un toxique chez un patient pour expliquer un état comateux pour lequel l'étiologie n'est pas connue,

3) à cause de raisons médicolégales,

4) une thérapeutique spécifique doit être mise en oeuvre.

\section{Évaluation de la gravité}

L'évaluation de la gravité est essentiellement anamnestique. Un interrogatoire soigneux (heure présumée 
d'ingestion, toxiques présents au domicile) doit reconstituer l'histoire de l'intoxication et confirmer son caractère accidentel. Parmi les produits les plus dangereux sont à signaler les digitaliques, les antidépresseurs, la colchicine, la nivaquine, la théophylline, le métaldéhyde, le paraquat, l'eau de javel concentrée, le monoxyde de carbone, les hydrocarbures et tous les solvants ou la soude caustique. L'aspirine présentée dans un conditionnement pour adulte peut être très dangereuse pour le jeune enfant. La notion d'une intoxication volontaire est un critère de gravité en soi et doit faire craindre une intoxication polymédicamenteuse. Les scores de gravité prédictifs n'ont pas été validés chez l'enfant en France.

\section{Particularités pédiatriques du traitement}

\section{Intoxications graves}

La prise en charge de l'enfant est identique à celle de l'adulte. Elle repose sur le maintien des fonctions vitales, sur le traitement symptomatique et sur les techniques d'épuration : épuration digestive, diurèse forcée, épuration extrarénale, antidotes et chélateurs et ventilation pour les toxiques volatils (Tableau VI).

En 1992, l'épuration digestive à fait l'objet d'une conférence de consensus de la Société de Réanimation de langue française (32).

Les indications actuelles du lavage gastrique sont les carbamates, les antidépresseurs tricycliques, les quinidiniques, et les digitaliques (32).

Le charbon activé inhibe l'absorption et augmente la clairance de nombreuses substances (33). Son efficacité parait supérieure à celle du lavage gastrique et des vomissements provoqués (34). Une administration répétée peut être proposée dans les intoxications par les antidépresseurs tricycliques ou les digitaliques. Les alcools, les métaux lourds, les hydrocarbures et les cyanures ne sont pas adsorbables (10)

L'association du lavage gastrique à l'administration de charbon activé n'a pas fait la preuve de sa supériorité par rapport au charbon activé seul (35).

Les indications de l'épuration extrarénale (EER) en toxicologie concernent des substances de petit poids moléculaire, de faible volume de distribution et peu lié aux protéines, la clairance d'épuration doit être supérieure à la clairance corporelle totale spontanée, et l'élimination du toxique doit diminuer significativement les risques pour le malade (36). Les toxiques pour lesquels l'indication d'EER est formellement reconnue sont l'alcool méthylique, les glycols, les salicylés et la théophylline (36).
Tableau VI : Fréquence d'utilisation des différents moyens d'épuration chez l'enfant.

\begin{tabular}{|c|c|}
\hline Technique depuration & Frequence dfutilisation \\
\hline $\begin{array}{l}\text { Épuration digestive } \\
\text { Charbon activé } \\
\text { Ipéca } \\
\text { Apomorphine } \\
\text { Lavage gastrique } \\
\text { Irrigation intestinale }\end{array}$ & $\begin{array}{l}\text { Très fréquente } \\
\text { Fréquente } \\
\text { Nulle } \\
\text { Peu fréquente } \\
\text { Exceptionnelle (intoxication par le fer) }\end{array}$ \\
\hline $\begin{array}{l}\text { Diurèse forcée } \\
\text { Neutre } \\
\text { Alcaline }\end{array}$ & $\begin{array}{l}\text { Nulle } \\
\text { Nulle }\end{array}$ \\
\hline $\begin{array}{l}\text { Épuration extrarénale } \\
\text { Dialyse péritonéale } \\
\text { Hémodialyse } \\
\text { Hémofiltration } \\
\text { Exsanguinotransfusion }\end{array}$ & $\begin{array}{l}\text { Exceptionnelle } \\
\text { Nulle } \\
\text { Nulle } \\
\text { Nulle }\end{array}$ \\
\hline $\begin{array}{l}\text { Antidotes } \\
\text { Flumazénil } \\
\text { Hypochlorite de sodium }\end{array}$ & $\begin{array}{l}\text { Exceptionnelle } \\
\text { Nulle }\end{array}$ \\
\hline
\end{tabular}

La dialyse péritonéale est la méthode d'EER de choix en pédiatrie du fait de sa facilité d'emploi (37). La supériorité de l'hémofiltration n'est pas démontrée (36).

L'intoxication par les antidépresseurs avec troubles du rythme ou de la conduction fait appel en première intention à l'alcalinisation par du lactate de sodium molaire, administré rapidement par voie intraveineuse (10).

Deux antidotes peuvent avoir un intérêt dans la prise en charge des comas d'origine toxique : le flumazénil $\left(\right.$ Anexate $\left.{ }^{\circledR}\right)$ et la naloxone (Narcan $\left.{ }^{\circledR}\right)(10)$. Ils permettent le réveil des comas induits respectivement par les benzodiazépines et les opiacés, mais leur utilisation est considérablement limitée par leur demi-vie très brève et leurs contre-indications. Ils peuvent également servir de test diagnostique (38).

La $\mathrm{N}$-acétyl-cystéine est l'antidote du paracétamol. Son indication est fonction de la paracétamolémie à la $4^{\text {inc }}$ heure [nomogramme de Rumack et Matthew (21)].

En cas d'intoxication au monoxyde de carbone, l'oxygénothérapie hyperbare est indiquée en cas de perte de conscience initiale ou de signe clinique objectif (trouble de conscience, syndrome d'irritation pyramidale, convulsions, hypotonie). Le taux sanguin d'HbCO n'est pas un critère absolu lorsqu'il est pris isolement (39).

Le Tableau VII compare les données du Centre Antipoison de Lille sur les techniques d'épuration utilisées en 1995 et en 1999. 
Tableau VII : Technique d'épuration, données Centre Antipoison de Lille. Comparaison entre 1995 et 1999 (traitement réel à l'hôpital).

\begin{tabular}{|l|c|c|}
\hline Épuration digestive & 1995 & 1999 \\
\hline Charbon & $21 \%$ & $17 \%$ \\
Ipeca & $6 \%$ & $1 \%$ \\
Apomorphine & $0 \%$ & $0 \%$ \\
Lavage gastrique & $8 \%$ & $5 \%$ \\
Irrigation intestinale & $0 \%$ & $0 \%$ \\
\hline Diurèse forcée & $1 \%$ & NS \\
\hline Épuration extrarénale & & \\
\hline Dialyse péritonéale & 0 & 0 \\
Hémodialyse & 0 & 0 \\
Hémofiltration & 0 & 0 \\
Exsanguinotransfusion & 0 & 0 \\
\hline Antidotes & & \\
\hline Anexate & NS & NS \\
N acétyl cystéine & NS & NS \\
Cyanokit & 0 & NS \\
Narcan & 0 & NS \\
Vit K & NS & NS \\
\hline
\end{tabular}

\section{Intoxications bénignes}

En cas d'intoxication accidentelle bénigne, les conseils donnés par les CAP permettent aujourd'hui d'éviter de nombreuses hospitalisations inutiles, mais il est probable qu'un plus grand nombre d'intoxications accidentelles pédiatriques pourraient bénéficier d'un conseil médical simple et d'une prise en charge ambulatoire.

Le lavage gastrique est traumatisant, il n'est pas justifiée en cas d'intoxication bénigne (32). Il expose au risque d'inhalation et n'a aucune vertu pédagogique.

L'administration de sirop d'ipéca a longtemps été la seule méthode simple d'épuration digestive utilisée. Son efficacité est d'autant plus grande qu'il est administré précocement, Les vomissements commencent 20 minutes après administration (40). Vingt huit pour cent des enfants vomissent après une dose de $15 \mathrm{~mL}$ et $99 \%$ après deux doses (40). Il doit être administré dans l'heure suivant l'intoxication (35) ; ceci impliquerait qu'il soit pris à domicile. La conférence de consensus de la SRLF n'en recommandait d'ailleurs pas la présence à domicile (32). Les CAP considèrent son utilisation à domicile, sans surveillance médicale, comme dangereuse, vu la toxicité de ce sirop.

En France, le charbon activé est disponible sous forme de poudre de charbon activé officinal (Carbomix ${ }^{\circledR}$ ). Administré en dose unique, le charbon est utilisable dans les intoxications paucisymptomatiques, et semble moins traumatisant que les vomissements provoqués.
Cependant son efficacité en termes de morbidité et de mortalité n'a pas été démontrée (10).

\section{Prévention}

Les intoxications du jeune enfant entrent dans le cadre des accidents à domicile et relèvent à ce titre des campagnes de prévention générale $(8,41)$. L'effet de ces campagnes est difficile à évaluer (41) et elles doivent être complétées par des actions de prévention individuelle de la part des médecins (42), en particulier lors des consultations systématiques ou en présence d'un enfant victime d'intoxication.

La concertation entre l'industrie pharmaceutique et la direction générale de la santé a abouti à la réduction de la quantité de produit actif par conditionnement. La généralisation des conditionnements de sécurité (plaquettes monoalvéolaires therrnosoudées, comptegouttes solidaires des flacons, bouchons de sécurité) permet d'éviter de nombreuses intoxications d'enfants et d'en diminuer la gravité. En juillet 1981, l'introduction d'un bouchon de sécurité sur les flacons de déboucheur liquide (Destop ${ }^{\circledR}$ ), le changement de couleur du produit, l'introduction d'ammoniaque comme répulsif et un étiquetage plus informatif ont permis une réduction de $35 \%$ des appels concernant ce produit (8). En 1989, la généralisation des bouchons de sécurité a eu le même effet sur les intoxications par les poudres pour lave-vaisselle (8). Il faut signaler le rôle de surveillance et d'alerte des CAP sur les risques toxiques des produits.

La démarche éducative envers la famille des enfants intoxiqués fait partie intégrante de leur prise en charge. Cette démarche éducative n'exclut par le signalement des cas où l'intoxication entre dans le cadre d'une carence de soins manifeste. Le Tableau VIII donne les mesures de prise en charge et de prévention et des organismes fournissant des documents pédagogiques. Il faut insister sur l'importance des CAP qui ont un rôle dans le conduite à tenir devant une intoxication aiguë chez l'enfant, en réduisant les admissions inutiles dans les services d'urgence et en éduquant le public. Les CAP ont une mission d'éducation et de prévention de la population. Ils diffusent des plaquettes, des articles de presse et participent à la formation de relais (enseignants, professionnels de la santé). Des sites Internet spécifiques pour l'éducation du grand public sont répertoriés dans le Tableau IX.

\section{Conclusion}

La majorité des intoxications concerne des patients d'âge pédiatrique. Les intoxications accidentelles du jeune enfant sont le plus souvent bénignes, et les intoxications volontaires de l'adolescent sont plus 
graves. Les intoxications de l'enfant ont un pronostic moins sévère que les intoxications de l'adulte, à condition de repérer rapidement par l'interrogatoire et l'examen clinique les rares cas graves qui relèvent d'une prise en charge comparable à celle de l'adulte. Le traitement des intoxications paucisymptomatiques, le plus approprié semble être l'administration d'une dose unique de charbon activé, suivie d'une surveillance médicale de quelques heures. Les recherches toxicologiques ne doivent pas être systématiques, mais elles doivent être mises en oeuvre dans le cadre d'une étiologie inconnue, pour des raisons médicolégales ou pour mettre en oeuvre une thérapeutique spécifique. La prévention par la réduction des risques des produits laissés à la portée des enfants doit être une préoccupation importante. Il est nécessaire que les professionnels de santé détectent et signalent les cas d'intoxications au centre de toxicovigilance, afin que des actions de prévention puissent se mettre en place (information, modification de formule ou de présentation).

Tableau VIII : Principaux points à enseigner aux parents pour la prévention et la prise en charge des intoxications d'enfants (10).

\begin{tabular}{|l|l|}
\hline \multicolumn{1}{|c|}{ Mesures préventives } & \multicolumn{1}{c|}{ Mesures de prise en charge initiale } \\
\hline $\begin{array}{l}\text { Ranger les médicaments en cours d'utilisation dans une } \\
\text { armoire à pharmacie verrouillée }\end{array}$ & Réagir dès la suspicion d'intoxication \\
\hline $\begin{array}{l}\text { Séparer les médicaments pour enfant des médicaments } \\
\text { pour adulte }\end{array}$ & $\begin{array}{l}\text { Ne pas faire vomir. Ne pas administrer de lait ou un } \\
\text { quelconque antidote. Laisser l'enfant à jeun }\end{array}$ \\
\hline Rapporter les médicaments inutiles au pharmacien & $\begin{array}{l}\text { Se munir des éléments pouvant aider à l'identification } \\
\text { du toxique (emballage, notice) }\end{array}$ \\
\hline Éviter l'automédication & $\begin{array}{l}\text { Prendre conseil par téléphone auprès du médecin traitant } \\
\text { du centre antipoison ou du centre 15 }\end{array}$ \\
\hline $\begin{array}{l}\text { Ne pas préparer les médicaments de la journée dans un } \\
\text { pilulier accessible }\end{array}$ & \begin{tabular}{l} 
Laver à l'eau claire les téguments exposés à un caustique \\
\hline $\begin{array}{l}\text { Stocker lés caustiques et les produits d'entretien dans } \\
\text { un placard fermé à clef. Ne pas les déconditionner }\end{array}$
\end{tabular} \\
\hline
\end{tabular}

Tableau IX : Sites INTERNET.

\begin{tabular}{|l|l|}
\hline Centre du Québec : centre de Toxicologie du Quebec & http ://www.ctq.qc.qa \\
\hline Aapcc : American Asscf Poison Central Centers : (USA) & www.aapcc.org/ \\
\hline $\begin{array}{l}\text { NJPIES : New Jersey Poison Information and } \\
\text { Education system web site : (site sur le CO) (USA) }\end{array}$ & http ://njpies.org/co/index.htm \\
\hline CAP de Lille & http ://www.chru-lille.fr/cap/lille.htm \\
\hline Alabama : (Éducation pour les différents âges, objets dangereux) & www.alapoisoncenter.org/ \\
\hline Californias Health care System & http ://www.carolinas.org/services/poison \\
\hline
\end{tabular}

\section{Références}

1. Lavaud J. Les accidents domestiques. Pédiatr. Prat. $1992 ; 40: 1-6$.

2. Unité INSERM SC8. Statistiques de mortalité par accidents de 0 à 19 ans en 1994. La lettre du C.I.R.P.A.E. 1997 ; 41 : 4-6.

3. Blaho K., Winberg S., Merigian K. Pharmacological considerations for the pediatric patient. Optom Clin. $1996 ; 5: 61-90$
4. Graeter L.J., Mortensen M.E. Kids are different : developmental variability in toxicology. Toxicology. 1996 ; $111: 15-20$.

5. Litovitz TL., Klein-Schwartz W., Caravati EM., Youniss J., Crouch B., Lee S. 1998 annual report of the American Association of Poison Control Centers Toxic Exposure Surveillance System. Am. J. Emerg. Med. 1999 ; 17 : 435-487.

6. Prachelroad F., Clark RF., Dean B., Krenzelok EP. Treated vs reported toxic exposure discrepancies between a poison control center and a member hospital. Vet. Hum. Toxicol. $1990 ; 32: 156-9$. 
7. Shannon M. Ingestion of toxic substances by children. New Eng. J Med. 2000; 342 : 186-191.

8. Lavaud J. Intoxications aiguës de l'enfant. Encycl. Méd. Chir. (Elsevier, Paris) Pédiatrie. 4125 A151992.

9. Mathieu-Nolf M. Rapport d'activité du Centre anti-poison des régions Nord, Pas-de-Calais, Picardie et Haute Normandie. Centre anti-poison de Lille, bd Oscar Lambret. 59037 Lille, 1995-1999.

10. Cremer R., Leclerc F., Leteurtre S., Martinot A. Particularités des intoxications de l'enfant. Intoxications aiguës (Jaeger A., Vale JA. Eds) Elsevier Paris. 1999 ; pp 468-82.

11.Dine MS., McGovern ME. Intentional poisoning of children. An overlooked category of child abuse : report of seven cases, and revue of the literature. Pediatrics 1982 ; $70: 32-5$

12. Gosset D., Hédouin V., Ruvuelta E., Desurmont M. Les sévices d'action. Dans maltraitance à enfants. Masson, Paris. 1995 ; pp 11-60.

13. McClure RJ., Davis PM. Meadow SR., Sibert JR. Epidemiology of Münchausen syndrome by proxy, nonaccidental poisoning, and non-accidental suffocation. Arch. Dis. Child. 1996 ; 75 : 57-61.

14.Le Heuzey MF., Isnard P., Badoual AM., Dugas M. Enfants et adolescents suicidants. Arch. Pediatr. 1995 ; $2: 130-5$.

15. Center for disease control. Fatal and nonfatal suicide attempts among adolescents. Oregon, 1988-1993. Morb. Mortal Wkly Rep. 1995 ; 44 : 312-5, 321-3.

16. Hawton K., Fagg J. Deliberate self poisoning and self injury in adolescents. A study of characteristics and trends in Oxford. 1976-89. J. Psychiatry $1992 ; 161: 816-23$.

17. Commitee on adolescence. American Academy of Pediatrics. Suicide and suicide attempts in adolescence and young adults. Pediatrics $1988 ; 81: 322-4$

18. O'Malley PM., Johnston LD., Bachman JG. Adolescent substance use. Epidemiology and implications for public policy. Pediatr. Clin. North Am. 1995 ; 42 : 241-60.

19. Linke JC., Mathieu-Nolf M. Computer as an aid to identify drugs. Third Meeting on computer as an aid in Poison Centers, Lille 9-12 December, 1998.

20. Sugarman JM., Rodgers GC., Paul RI. Utility of toxicology screening in a pediatric emergency departement. Ped. Emerg. Care. 1997 ; 13 : 194-7

21. Rumack BH., Matthew H. Acetaminophen poisoning and toxicity. Pediatrics $1975 ; 55: 871-6$.

22. Dehon B., Lhermitte M. Cyanures et anions. Dans Toxicologie et pharmacologie médicolégales (Ed Kintz P.), Elsevier Paris 1998 : pp147-88.

23. Soliman SA., Sovocool GW., Curley A., Ahmed NS., El Fiki S., El Sebae AK. Two acute human poisoning cases resulting from exposure to diazonon transformation products in Egypt. Arch. Environ. Health 1982 ; 37 : 207-12.

24. Mura P., Piriou A. Cannabis. Dans Toxicologie et pharmacologie médicolégales (Ed Kintz P.), Elsevier Paris 1998 : pp543-54.

25.Belson MG., Simon HK., Sullivan K., Geller RJ. The utility of toxicologic analysis in children with suspected ingestions. Pediatr. Emerg. Care 1999 ; 15 : 383-7.
26. Belson MG., Simon HK. Utility of comprehensive toxicologic Screens in children. Am. J. Emerg. Med. 1999; $17: 221-4$.

27.Labat L., Deveaux M., Dubost J.P. Applications de l'électrophorèse capillaire en toxicologie clinique et médicolégale. Ann. Toxicol. Anal. 2000 ; 12 : 179-195.

28. White M.A. A comparizon of inductively coupled plasma mass spectrometry with electrothermal atomic absorption spectrophotometry for the determination of trace elements in blood and urine from non occupationally exposed populations. J. Trace Elem. Med. Biol. 1999 ; 13 : 93-101.

29. Blake K.V., Bailey D., Zientic G.M., Hendeles L. Death of a child associated with multiple overdoses of acetaminophen. Clin. Pharmacol. 1988 ; 7 : 391-397.

30. Hanzlick R. National Association of Medical Examiners Pediatric Toxicology. Registry Report 3 case submission summary and data for acetaminophen, benzene, carboxyhemoglobin, dextrometorphan, ethanol, phenobarbital and psudoephedrine. Am. J. Forensc Med. Pathol. 1995; $16: 270-277$.

31. Mahoney JD., Gross PL., Stern TA. Quantificative serum toxic screening in the management of suspected drug overdose. Am. J. Emerg. Med. 1990 ; 8 : 16-22.

32.Jury de consensus : Lejonc J., Elkharrat D., Lapandry C., Leblanc JP., Robert R., Saint-Martin J., Simon D., Taburet AM. Epuration digestive lors des intoxications aiguës. Réan. Urg. 1993 ; 2 : 169-75.

33. Levy G. Gastrointestinal clearance of drugs with activated charcoal. N. Engl. J. Med. 1982 ; 307 : 676-8.

34. Lheureux P., Askenasi R. Prévention de l'absorption digestive des toxiques : une réévaluation clinique. Deuxième partie : utilisation du charbon activé et des laxatifs. Réan. Urg. $1992 ; 1$ : 915-26.

35.Leclerc F., Closset M., Hue V., Martinot A. Comparaison de l'efficacité des différentes méthodes d'épuration digestive chez l'enfant. Réan. Urg. 1993 ; 2 : 229-32.

36. Tirot P., Harry P., Bouachour G., Alquier P. Epuration extrarénale en toxicologie. Réan. Urg. 1993 ; 2 : 27-37.

37. Gouyon JB., Cochat P., Epurations extrarénales en réanimation pédiatriques. Rev. Prat. 1994 ; 44 : 1353-7.

38.Lacroix J., Gaudreault P., Nantel AJ. Intoxications : généralités. Dans Urgences et soins intensifs pédiatriques (Lacroix J., Gauthier M., Beaufils F., eds), Paris : Doin ; 1994 : pp801-29.

39. Myers RA., Britten JS Are arterial blood gases of value in treatment decisions for carbon monoxide poisoning? Crit. Care Med. 1989 ; 17 : 139-142.

40. American Academy of Clinical Toxicology, European Association of Poisons Centers and Clinical Toxicologists. Position statement : ipec syrup. J. Toxicol. Clin. Toxicol. $1997 ; 35: 699-709$.

41. Bass JL., Christoffel KK., Widome M. Childhood injury prevention conseling in primary care settings : a critical review of the literature. Pediatrics $1993 ; 92$ : 544-50.

42. Baudier F., Janvrin MP., Lévèque B. Les accidents de l'enfant : étude sur la place des médecins dans la démarche de prévention. Dans les Journées parisiennes de pédiatrie 1994, Paris, Flammarion Médecine-Sciences : 1994, pp 133-9. 